\title{
CORRIGENDUM
}

\section{Green tea and coffee consumption is inversely associated with depressive symptoms in a Japanese working population - CORRIGENDUM}

Ngoc Minh Pham, Akiko Nanri, Kayo Kurotani, Keisuke Kuwahara, Ayami Kume, Masao Sato, Hitomi Hayabuchi and Tetsuya Mizove

First published online 9 July 2013

doi: 10.1017/S1368980013000360, published online by Cambridge University Press 4 March 2013

The authors would like to amend an incorrect figure given in table 1: Participants' characteristics according to green tea and coffee consumption: men and women ( $n$ 537) aged 20-68 years from two workplaces in north-eastern Kyushu, Japan, 2009

In the column Green tea consumption: $\geq 4 \mathrm{cups} / \mathrm{d}$ ( $n$ 97). The $\%$ value for Physical activity ( $\geq 5$ MET-h/week) $\S$ should be corrected to $37 \cdot 1$ rather than $3 \cdot 0$

\section{Reference}

Pham NM, Nanri A, Kurotani K, Kuwahara K, Kume A, Sato M, Hayabuchi H \& Mizoue T (2013) Green tea and coffee consumption is inversely associated with depressive symptoms in a Japanese working population. Public Health Nutrition, published online 4 March 2013, doi:10.1017/S1368980013000360 